\title{
Characterization of Off Stoichiometric Silicon Oxide by Thermo, Cathode, and Photo- Luminescence
}

\author{
By Carlos Ruiz* \\ Rosa Lopez-Estopier ${ }^{+}$ \\ Jesus Alarcon-Salazar \\ Marco A. Vasquez- $A^{*}$ \\ Dainet Berman $\neq$ \\ Tom M. Piters † \\ Jorge Pedraza" \\ Mariano Aceves-Mijares"
}

\begin{abstract}
Off Stoichiometric Silicon Oxide, or Silicon Rich Oxide (SRO), obtained by LPCVD was studied by Photo, Cathode and Thermo luminescence. The Silicon excess in the SRO films varied between 2 and $12 \%$ at. In the deposited samples, the luminescence was observed in the blue region by all techniques. This is an indication that the high temperature used during the deposit produces point defects. After annealing at 1100 ${ }^{\circ} \mathrm{C}$ in nitrogen during 3 hours, the blue emission is still observed suggesting that the nanopoints responsible of the blue emission are not affected. However, after annealing, the photoluminescence of SRO 30, with $5 \%$ of silicon excess, shows drastic increase of the red emission contrarily to the cathodoluminescence, which, after annealing the blue emission, has a big increment. This contradictory observation cannot be explained as a simple emission of oxide defects, rather a mixed model that includes the effect of charge trapping characteristic of the SRO and coulombic interaction, is proposed. Still, more work has to be done to have a detailed mixed model that predicts the emission of the SRO films obtained by LPCVD.
\end{abstract}

Keywords: LPCVD, Luminescence, Silicon Rich Oxide

\section{Introduction}

Silicon Rich Oxide (SRO), or off stoichiometric silicon oxide, is a multiphase material formed by $\mathrm{SiO}_{2}, \mathrm{SiO}_{\mathrm{x}}$ and elemental silicon. The $\mathrm{SRO}$ can

\footnotetext{
* PhD Student, University of Sonora, Mexico.

${ }^{+}$Professor (Catedrática Conacyt), Autonomous University of San Luis Potosí, Mexico.

${ }^{\circ} \mathrm{PhD}$ Student, National Institute of Astrophysics, Optics and Electronics, Mexico.

- Posdoctoral Fellow, National Institute of Astrophysics, Optics and Electronics, Mexico.

* Professor, University of Sonora, Mexico.

${ }^{\dagger}$ Professor, University of Sonora, Mexico.

- Professor, National Institute of Astrophysics, Optics and Electronics, Mexico.

* Professor, National Institute of Astrophysics, Optics and Electronics, Mexico.
} 
be obtained by various deposition techniques. However Low Pressure Chemical Vapor Deposition (LPCVD) is a simple technique of obtaining it, and allows varying the silicon excess with relative precision in a simple way. The silicon excess in the SRO obtained by the LPCVD is controlled by the ratio of the partial pressure of reactive gases:

$$
R_{0}=\frac{\mathrm{N}_{2} \mathrm{O}}{\mathrm{SiH}_{4}}
$$

From 0 to $17 \%$ of silicon excess is obtained for Ro from 100 to 3 (Dong et al., 1978). Depending on the silicon excess, structural, optical and electrical characteristics vary. SRO presents high Photoluminescence (PL), especially that obtained by LPCVD. However, silicon excess between 8 and $4 \%$ shows a high emission, but this range also shows low conductivity and low density of silicon nanocrystals (Sinc). On the other hand, silicon excess of $12 \%$ approximately or higher has many nanocrystals, higher conductivity and reduced emission. To explain the photo emission, researchers employ either the quantum confinement emission theory or the emission due to oxide defects. However, up to present time, there exists no model that satisfactorily explains and predicts all the details of the emission obtained in SRO.

The SRO is a material rich on electronic charge traps. A density of traps in the order of $10^{17} \mathrm{~cm}^{-3}$ in Ro 30, has been reported (Aceves et al., 1996). It is known that SRO gains or losses one electron in each trap. That is, SRO can trap positive or negative charge. Then, the effect of the charge on the emission of light has to have an important role, especially if the coulombic interaction between positive and negative traps aids to the decay of electrons, resulting in the emission.

The Photoluminescence is a very well-known characterization technique, and basically consists of the injection of photons of relative low energy into the sample under study. Normally, this technique can be used to obtain the emission and the absorption as a function of the wavelength. Details on this technique can be seen elsewhere (Shinde et al., 2012; Pankove, 1971). This technique has been used to study the characteristic emission of SRO.

In the case of cathode luminescence (Yacobi and Holt, 1990), the samples are subjected to a beam of high energy electrons, instead of photons as in PL. Most of the authors manage the CL as a PL emission but with higher energy particles, without considering that electros have charge and that they could influence the emission of light. In spite that, $\mathrm{CL}$ is a technique that can be used to better understand the PL emission, and there are not many works on the CL of SRO.

On the other hand, Thermoluminescence (TL) is a luminescence characteristic of some materials after they are exposed to ionizing radiation (Richter, 2007). Energetically excited electrons move to traps produced by the radiation, or to traps already existing in the material. Afterwards, heating the sample, the trapped electrons are released to their ground states and in this process; emission of characteristic photons could be emitted. To our knowledge, our group is the only one that is studying the TL of SRO. 
As known, annealed SRO presents high red photoluminescence, but almost no blue photoluminescence. On the contrary, cathode luminescence shows high blue luminescence and small intensity red emission, see Figures 1 and 2. This contradiction has not been explained.

In this paper, in order to contribute to better understanding the light emission in SRO, besides of the photoluminescence (PL), cathode and thermo luminescence (CL and TL) were studied. SRO samples with silicon excess varied from 2 to $12 \%$ approximately and were prepared by LPCVD. Samples as deposited and annealed at high temperature were studied. Also, a model that explains the red and blue photo and cathode luminescence is proposed.

\section{Experimental Procedure}

Silicon Rich Oxide films were deposited by LPCVD on P type, 3-5 ohm $\mathrm{cm}$, silicon substrates at $730^{\circ} \mathrm{C}$. A homemade LPCVD hot wall system was used. The reactive gases were Nitrous Oxide and 5\% Silane in nitrogen. SRO films of $\mathrm{R}_{0}=40,30,20 \mathrm{y} 10$ were fabricated, corresponding to a silicon excess from 2 to $12 \%$, approximately. Once the deposited some samples were annealed at $1100{ }^{\circ} \mathrm{C}$, during 180 minutes in nitrogen. The average thickness was $80 \mathrm{~nm}$. To obtain TL, SRO films were activated using beta radiation with a $0.055 \mathrm{~Gy} / \mathrm{s}$ dose, and the total radiation was 100, 200, 400 and $800 \mathrm{~Gy}$. The equipment used was a TL-DA-15 by Riso National Laboratories, Denmark that includes a Sr-90 beta source, and using color filters it was determined that the emission was in the blue region. The heating rate during the readout was 2 ${ }^{\circ} \mathrm{C} / \mathrm{s}$. The PL was obtained with a Horiba Fluoro-max 3. All samples were excited with a wavelength of $300 \mathrm{~nm}$ and the detector was swept from $370 \mathrm{~nm}$ to $1000 \mathrm{~nm}$. CL was obtained with a luminoscope model ELM2-144 with $5 \mathrm{kV}$ acceleration voltages and $0.3 \mathrm{~mA}$ emission current pumps the samples, and the fluorometer records the emission spectra.

\section{Results and Discussion}

Figure 1 (a) and (b) show the photoluminescence of the different films before and after annealing at $1100{ }^{\circ} \mathrm{C}$ during 180 minutes in nitrogen. All as deposited samples emit basically around $420 \mathrm{~nm}$. However, some emission can be observed from 600 to $850 \mathrm{~nm}$. 
Figure 1. PL Emission of SRO with Ro 10, 20, 30 and 40, without (a) and (b) with Thermal Treatment at $1100{ }^{\circ} \mathrm{C}$ during 3 hours in Nitrogen, all of them Show Emission at around $420 \mathrm{~nm}$, Especially as Deposited Samples. (c) Excitation Curves of the Same Samples, the Detection Wavelength is shown in the Legend of the Figure

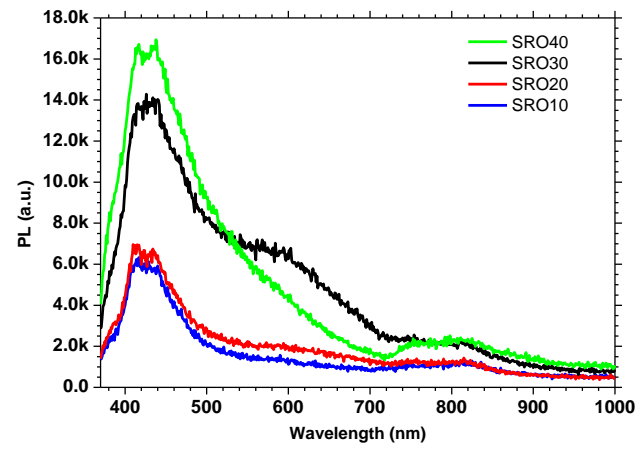

(a)

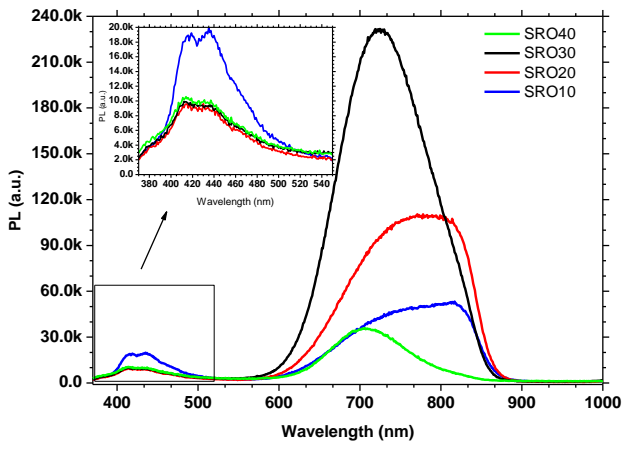

(b)

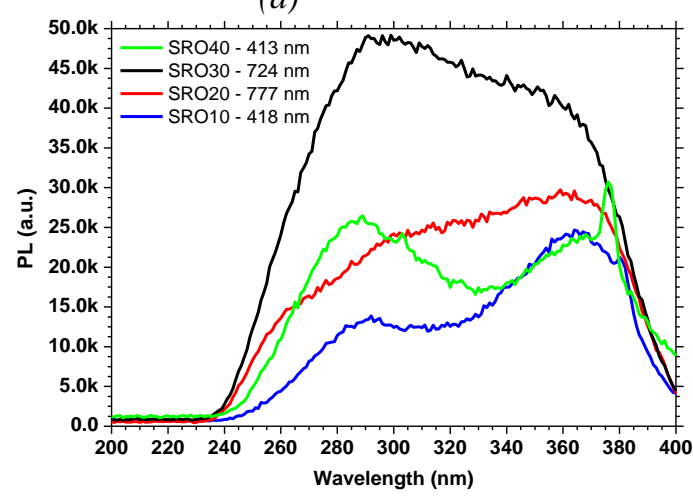

(c)

After annealing, a relative intense luminescence band emerges around 700 $\mathrm{nm}$ while the luminescence around $420 \mathrm{~nm}$ is little affected. The strongest increase of the red luminescence is seen in the sample with Ro 30 corresponding to a silicon excess of 5\% approximately. PL emission around $700 \mathrm{~nm}$ increases as the $\mathrm{R}_{0}$ increases, and SRO 30 reaches the higher intensity, after this value if the $\mathrm{R}_{0}$ is increased then the PL begins to reduce. So that, the SRO 30 has an optimum silicon excess of $5 \%$ approximately. These curves are typical of SRO obtained by LPCVD, and they emit mainly in the 600 to 850 $\mathrm{nm}$ range. The emission is ascribed principally to $\mathrm{SiO}_{2}$ defects as oxygen vacancies (Rodríguez et al., 2014). Defects as WOB (weak oxygen bond), NOV (non-oxygen vacancy), E defect, NBOHC (non-bridging oxygen hole center) and dangling bonds have been found in quartz using techniques such as electronic spin resonance. The emissions of such defects are in the range of $415,455,520-560,680-710$ and $750-1100 \mathrm{~nm}(2.99,2.73,2.39-2.22,1.83-$ $1.75,1.66-1.13 \mathrm{eV}$ ) respectively, clearly in the emission range of our samples. On the other hand, SRO 10 is rich in Sinc and it does not have so many defects, also the silicon excess of SRO $40(2 \%)$ is so small that the density of the defects is not expected to be as high as that of SRO 30. Figure 1 (c), shows the excitation curves for the different SRO films. These curves were obtained 
fixing the detector at a specific wavelength, and then the pumping wavelength excitation varied from 200 to $400 \mathrm{~nm}$. The curves show a wide absorption range.

Figure 2 (a) and (b) show the CL of samples before and after annealing, respectively. CL emits in a longer range as compares with PL: from 400 to 850 $\mathrm{nm}$. Before annealing, again the main emission is in the blue region around 455 $\mathrm{nm}$, and SRO 30 shows a clear band. SR0 20 and 10 shows these emissions but much less intense, and in particular for SRO 10 the peaks are narrow and the emission is very small as can be seen. As observed in samples without annealing, the PL emission is small indicating that as deposited samples have already some defects but their density is not as big as that of the annealed samples. This could be the reason of the small emission observed in Figure 2 (a).

Figure 2. Cathode-Excitation of SRO at $5 K V$ and $0.3 \mathrm{~mA}(a)$ Before and (b) After Thermal Annealing

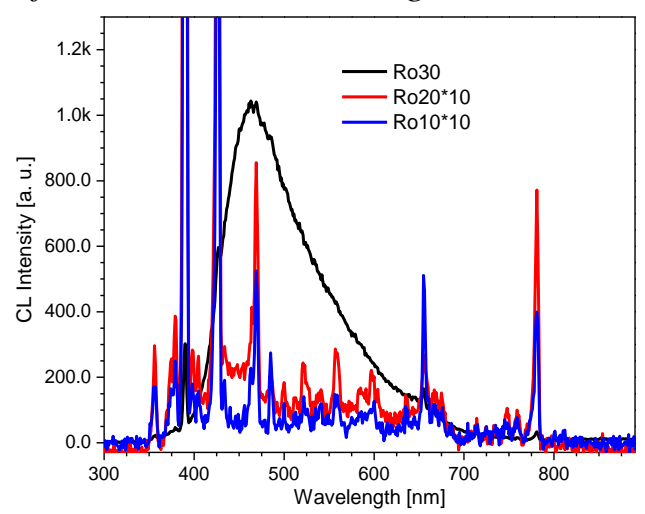

(a)

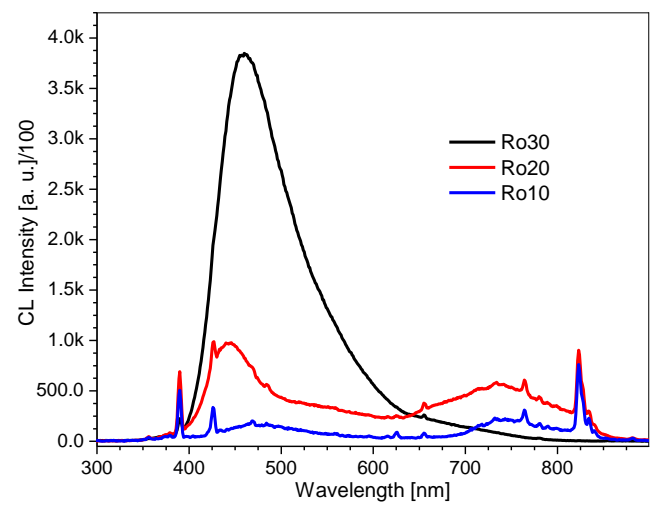

(b)

For CL, the higher emission is obtained in SRO 30 for both with and without annealing, as mentioned this time the larger peak arises in the range from 400 to $600 \mathrm{~nm}$, as can be seen in Figure 2. For annealed samples, as Ro reduces the peak in the range from 400 to $600 \mathrm{~nm}$ tends to be comparable of that one at 700 to $850 \mathrm{~nm}$, and as Ro increase the red peak tends to be negligible. Two important facts have to be mentioned in order to understand the CL emission. First, as the Ro increases the density of defects increases and the Sinc density reduces up to the case of Ro 30 where they do not exist. As mentioned, SRO is rich in electron traps and Ro 30 is the one that has the higher density. Then, when high cathode-electrons impinge in the film high energy excited electrons are produced, and these electrons reach higher energy traps that emit in the $400 \mathrm{~nm}$ range. Moreover, the cathode - electrons are charged particles that should produce an imbalance between positive and negative charge in equilibrium. So, it is likely that coulombic forces play an important role and they have to be included in the high energy photon emission. The PL and CL emits in the range of emission of defects that are present in the SRO, mentioned before. Either for PL or CL, the emission curves do not overlap that of excitation, meaning that a different mechanism produces 
the absorption and the emission. Nevertheless, the wide excitation spectrum could excite all the traps in the SRO, but for PL the energy is only able to excite mainly low energy mechanisms of emission. However, CL excites mainly high energy centers, but it is expected that both low and high energy centers would be excited.

Figure 3 (a) and (b) show the TL glow curves of a SRO film with 5\% silicon excess before and after thermal treatment. The irradiation dose was 550 Gy. The total TL intensity does not vary significantly before and after annealing. However, the composition of the glow curves shifts toward a somewhat more intense high temperature peak for the annealed sample. This reflects a change of the defect contents during the annealing of the SRO, as previously stated. Using an optical filter placed before the photomultiplier tube that collects the TL, it was possible to determine that the emission was also in the blue region. As in the case of CL and that observed for other researches and reviewed in the next paragraphs.

Trukhin et al. (2007) studied pure silica and with $0.015 \%$ wt silicon excess using thermoluminescence stimulated by $\mathrm{X}$ rays and $\mathrm{UV}$ radiation, they found a $2.7 \mathrm{eV}$ emission center, E' center, with a wide tail up to $4.5 \mathrm{eV}$. This center was corroborated by Electron Spin Resonance (ESR). In the same paper, the authors propose that the holes detrapped during heating of the sample could recombine with electrons due to coulombic attraction.

Buscarino et al. (2006) determined different $\mathrm{E}_{\alpha}^{\prime}, \mathrm{E}_{\delta}^{\prime}$ and $\mathrm{E}_{\gamma}^{\prime}$ point defects by ESR in amorphous silicon dioxide $\left(\mathrm{a}-\mathrm{SiO}_{2}\right)$. Defects were associated to positively charged oxygen vacancy: $\mathrm{O} \equiv \mathrm{Si} \bullet \mathrm{O} \equiv \mathrm{Si}+$ (where $\equiv$ represents the bonds to three oxygen atoms, $\bullet$ is an unpaired electron and + is a trapped hole), and the $\mathrm{E}_{\alpha}{ }_{\alpha}$ center consisting in a hole trapped in an oxygen vacancy.

Figure 3. Glow Curves for Ro 30 (a) Before and (b) After Thermal Treatment

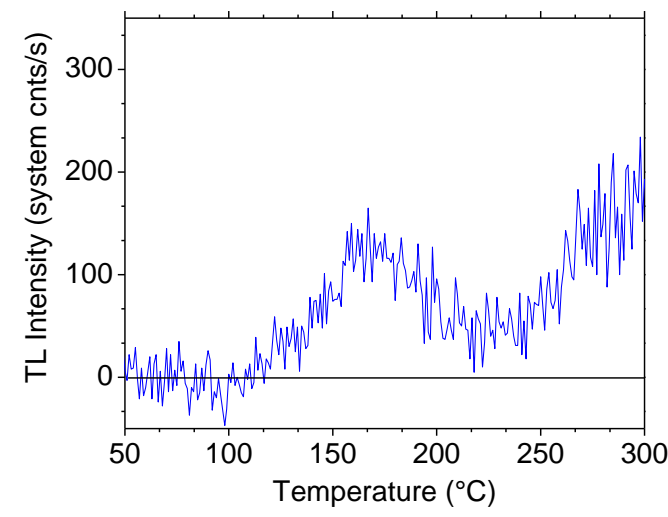

(a)

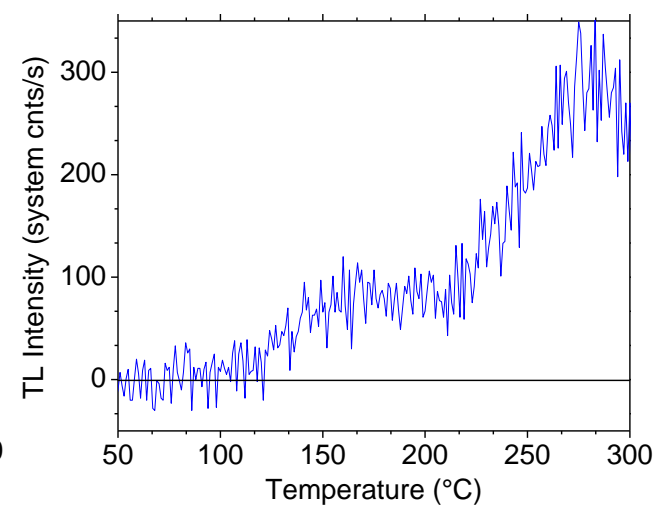

(b)

In a recent study, on silica nanoparticles prepared through a laboratory sol-gel technique (Spallino et al., 2014), the origin of the blue band has been associated with a defect pair consisting of a dioxasilyrane, $=\operatorname{Si}\left(\mathrm{O}_{2}\right)$, and a silylene, Siø. The author affirms that in experiments of thermochemical reactions have demonstrated the formation of oxygen related defects specific to the surface such as the nonbridging oxygen, $\mathrm{Si}-\mathrm{O} \cdot$, the dioxasilyrane, $\equiv \operatorname{Si}\left(\mathrm{O}_{2}\right)$, 
and the silanone, $\mathrm{Si}=\mathrm{O}$. They also relate the absorption at 3.69 and $3.85 \mathrm{eV}$ (336 and $310 \mathrm{~nm}$ ) with the emission peaks at 3.34, 3.17, 3.00 and $2.83 \mathrm{eV}(376$, $388,414,444 \mathrm{~nm})$.

Emission at $3.2 \pm 0.5 \mathrm{eV}$ in the UV-Blue thermoluminescence has been observed in $\mathrm{SiO}_{2}$ obtained by sputtering (Hickmott, 1972). In the aforementioned report, the authors correlate the optical absorption with the energy of a shallow trapping center. They correlate an absorption band from 2 to $8.5 \mathrm{eV}$ (620 to $165 \mathrm{~nm}$ ) with a trapping center $0.66 \mathrm{eV}$ down the conduction band for as sputtered films, and a trapping center at $0.84 \mathrm{eV}$ for $\mathrm{X}$ ray irradiated. They correlate these trapping centers with the $3.2 \pm 0.5 \mathrm{eV}$ emission band.

From the previous discussion, it is clear that the emission of silica-based materials has a common range of emission, and it is due to point defects that emit in the range from 400 to $860 \mathrm{~nm}$ ( 3 to $1.45 \mathrm{eV}$ ). These defects have been extensively studied and characterized. As can be observed from our results, the SRO is not an exception and the blue to near infrared emission is observed in films as deposited and annealed at high temperatures.

In the case of the SRO obtained by LPCVD, the high temperature during the deposition makes the material rich in $\mathrm{SiO}_{2}$ defects from the start. These defects are responsible for the observed PL emission in samples as deposited as shown in Figure 1 (a). The main emission is centered at around $420 \mathrm{~nm}$ coinciding with the emission reported by Spallino et al. (2014). It is then clear that the blue emission in PL is also due to defects (Spallino et al., 2014). When annealing takes place, a competitive process starts between the formation of silicon clusters, and the formation and accumulation of silicon-oxide defects. For a high percentage of silicon excess, say 12\% (SRO 10), the Si clusters dominate and the formation of Sinc is promoted instead of the defects, so low emission intensity is expected in all wavelengths. For small Si excess, say 5\% (SRO 30), new defects should be created and accumulated causing an intense emission in the whole range. However, for PL, the blue emission does not increase and the red emission presents a much higher intensity. For SRO 20 the characteristic is in between these extremes. From Figure 1 (b), it is clear that the emission centered at around 740 is increased as Ro increases, and the SRO 30 is the optimum. For all Ro's including SRO 10 the blue emission does not reduce, and then the results indicate that there is no change in the defects that produces the blue PL.

Figure 1(c) shows the excitation curves of the films. Spallino et al. (2014) report maximums at 336 and $310 \mathrm{~nm}$, and in spite of that we have blue emission our excitation curves do not show these maximum, instead we have a wide absorption band including the 336 and $310 \mathrm{~nm}$ wavelengths. The wide excitation band shown for these films corroborates that SRO is rich in different point defects.

Contrary to PL, the cathodoluminescence presents an intense blue emission, especially for annealed samples. Also, as Ro increases the blue emission increases, and the SRO 30 shows the higher emission. However, as Ro reduces the red and the blue emission intensities tend to be comparable. The 
intense blue CL emission cannot be due to the same defects observed in PL, because as stated their density is small. It is more likely that CL has to be related to the high energetic charged carriers impinging in the films.

Figure 4 shows the PL, CL and TL in the blue range. In this figure, the blue emission reported by Trukhin et al. (2007) is also included for comparison.

Figure 4. Normalized Spectra of CL, PL and TL of the SRO and Quartz, the TL of Quartz was Taken from (Trukhin et al., 2007).

\section{Energy [eV]}

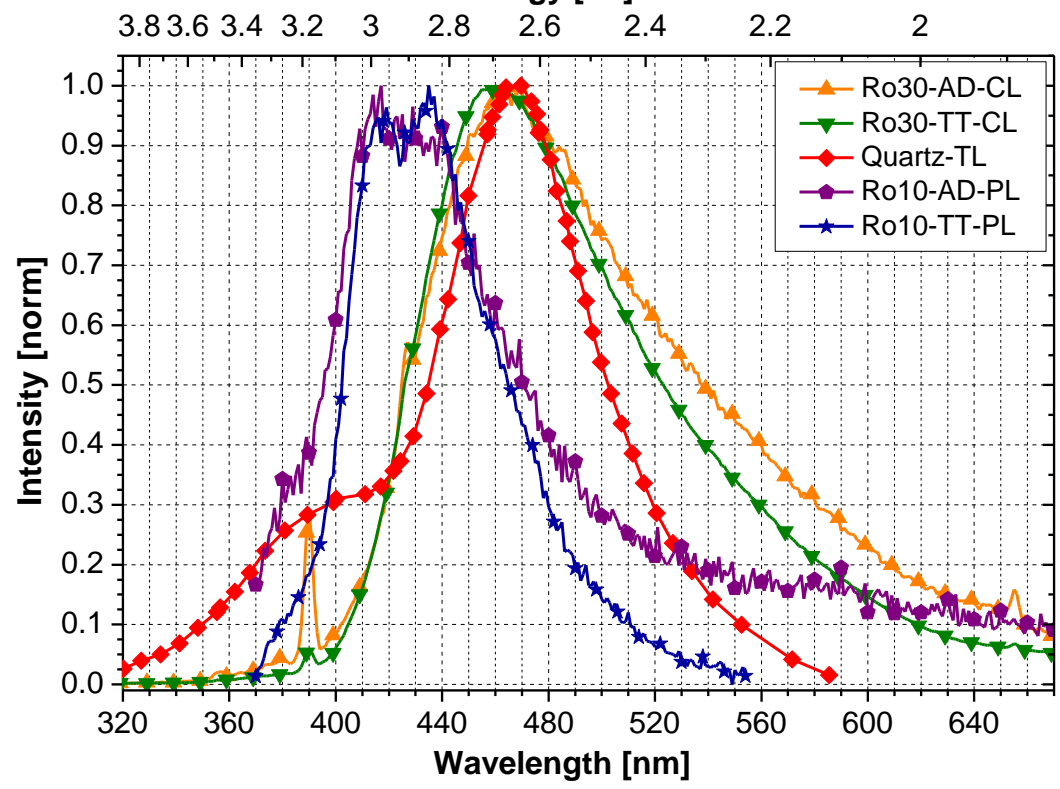

As mentioned, the fact that the blue emission occurs in deposited samples indicates that blue emission centers are created during the growth of the film at high temperatures by LPCVD. Also, observations of PL in the blue region for all Ro's indicate that the peak and the range of blue emission do not change with the thermal treatment, as seen in Figure 1 (a) and (b) and Figure 4. It is clear from figure 4 that two different groups of spectrum can be obtained, one for PL and other for TL and CL shifted to low energies. Moreover, all CL and TL spectrums are different of that of PL, meaning, again, that different emission mechanisms have to produce them.

Another important characteristic of the SRO is charge trapping. Being the same material the same defects that emit could act as acceptors or emitters of electrons. For example the vacancies of oxygen can trap holes or have unpaired electrons as discussed in the previous references. It is not difficult to think that when photons impinge into the SRO film some electrons will acquire enough energy to jump to another trap leaving the base state charge positive. Then, a charge imbalance will be produced. Even more, when high-energy ionizing radiation impinges on the films charged ions are produced. Then, high density of electrons and positive ions result inside the SRO film. A film rich in electros and ions will have a strong coulombic interaction. This will produce more 
energetic photons. That is, blue emission will be more probable than the red one.

The charge imbalance causes coulombic interaction between the charged traps. Trying to keep the mechanism of emission as simple as possible, the excited electrons decay between negative and positive traps due to coulombic attractions and this will produce the emission. This model is similar to that known as Donor Acceptor Decay, DAD (Yacobi and Holt, 1990; Richter, 2007) and is formulated as:

$$
E_{P L}=E_{g}-\left(E_{d}+E_{a}\right)+\frac{q^{2}}{4 \pi \varepsilon r}
$$

Where $E_{P L}$ is the emission energy of PL, $E_{d}$ and $E_{a}$ are the energies of the acceptor and donor pair; and $\varepsilon$ is the dielectric constant. The last term arises from the Coulombic interaction of the carriers and depends on the pair separation $r$.

In the past, our group using the DAD model proposes the distribution of the donor and acceptor pairs in SRO (López-Estopier et al., 2011), this result coincides with that proposed in (Spallino et al., 2014). In these references, it was found that the intramolecular allowed energy states and the decay in these states will produce some range of emission. That is, it has been demonstrated that the emission is due to the point defects that have specific emissions, however, this is not enough to explain the full range of emissions presented in SRO by the different excitation techniques, and neither to explain the small shifts observed.

Salh $(2011 \mathrm{a} ; 2011 \mathrm{~b})$ studied the formation of the Si-O agglomerates in SiOx subjected to thermal annealing. He and coworkers proposed that silicon atoms re-arrange due to heat, and could form small silicon rings given place to a cluster of different $\mathrm{SiOx}$ defects. Besides, they also propose the decay of electrons between two neighboring oxygen deficient centers. So, no matter the type of excitation, the excited molecules emit. However, in the presence of high density of charge, high coulombic interactions and then the decay between molecules inside the Si-O clusters have to be promoted. The combined emission, inside the molecules and between molecules, will produce the observed spectrum.

Then a combined model of emission of SRO is proposed, and it is based on the emission of the different point defects assisted by the coulombic forces. At the moment of writing this paper we are not able to give details on the formulation of this model, however a qualitative proposition is:

$$
E=E_{p d} \pm E_{C}
$$

Where, $E_{p d}$ is the point defect energy emission, and $E_{C}$ is the energy associated with the coulombic interaction.

Figure 4 shows the emission of TL, CL and PL of SRO and quartz in the blue region. As can be seen all of them are in the blue range, and their shape is quite similar in all the cases. The blue PL, both with and without annealing, is centered at $420 \mathrm{~nm}$. So, the same point defects have to be responsible for these emissions. Also, in PL an intense red emission is observed, due to the defect emission. In CL the more intense emission is in the blue side and it is centered 
on $460 \mathrm{~nm}$. The change in the wavelength intensity and the shift could be explained due to coulombic interactions. This could be explained as follows: when the sample is illuminated with UV light the electrons are excited by energy enough to produce emission between states inside of the molecule as that obtained in (Spallino et al., 2014). The low energy of the incident photons does not produce high coulombic interactions, and so most of the emission is due to the point defects. However, under electron bombardment of high ionizing energy, electrons are removed from their base states and many effects that produce charge imbalance take place promoting the emission due to centers inside the molecules and between molecules. However, due to the high density of the electronic charge present in films, a high imbalance between positive and negative traps causes strong coulombic attractions. So the electrons will have energy high enough to jump to other centers that will be able to produce emission by coulombic attraction in different wavelengths. Also, due to the high energy of cathode electrons, many charged particles than contribute to the coulombic decay, will be present. The higher the density of the traps, it is more probably that electrons reach traps in the right position to allow radiative emission, as in the case of SRO 30. The higher the excitation of the particles is, the higher the probability of excited electrons to decay at higher energies is, producing high-energy emissions. This explains that CL of RO 30 after the annealing emits principally in blue with a different peak as that of PL. After this discussion, the emission of SRO should be the sum of all of these possibilities, as stated in Eq. 3 and illustrated in Figure 5.

Figure 5. Mixed Model, (a) Decay Inside of Excited Nanopoint or Point Defect, (b) An Electron Trapped Producing a Negative Nanopoint, (c) A Hole Trapped Producing a Positive Nanopoint. The Arrows Indicate the Decay in such a System, the Solid Circle Indicates an Electron, the Circle Indicates a Hole and $r$ is the Distance between Traps. The Decay between (b) and (c) Will be More Probable if They Are Assisted by Coulombic Interaction. Si-O CLUSTER

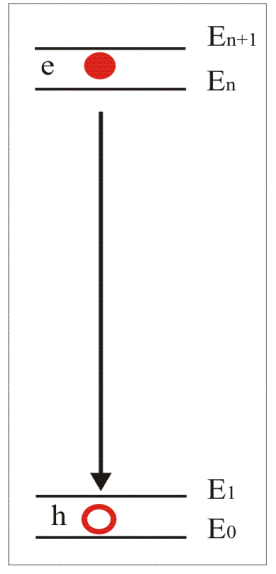

(a)

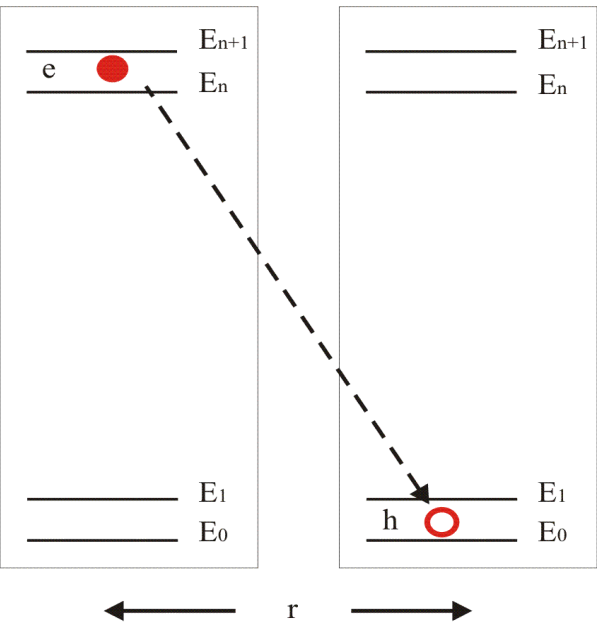

(b) (c) 
The PL of SRO 30 after annealing could be explained with similar arguments, but in this case because the excitation is of low energy the coulombic component allows only for low charge imbalance. Then, the red emission becomes dominant as seen in Figure 1(b). In the other case, high coulombic interactions will promote the blue emission over the red one.

\section{Conclusions}

SRO films with silicon excess from 2 to $12 \%$ were studied by Photo, Cathode, and Thermo - Luminescence. All of these techniques produce blue emission; however depending on the charge imbalance a red or blue emission is more probable. It is corroborated that the emission is due to silicon - oxide defects as that observed in other material whose matrix is $\mathrm{SiO}_{2}$ and reported in the literature. Special emphasis is paid for the blue emission that is very well understood and reported. Absorption and emission of the nanopoints, or point defects, has been studied and reports on the energy levels of the molecules can be found. However, from the analysis of the different luminescent emission of the SRO obtained by LPCVD, it is proposed that besides the intra nanopoints' emission, coulombic interaction between positive and negative nanopoints contributes to the emission spectrum. At the moment of writing this report, it is not possible to establish an analytical mixed model, and more work has to be done to obtain a model that calculates the emission spectrum of SRO obtained by LPCVD.

\section{References}

Aceves, M., Falcony, C., Reynoso-Hernandez., A., Calleja, W. and Torres, A. (1996) 'The Conduction Properties of The Silicon/Off-Stoichiometry-Si0 $0_{2}$ Diode', SolidState Electronics Vol. 39, No. 5, 637444. doi:10.1016/0038-1101(95)00174-3.

Buscarino, G., Agnello, S., and Gelardi, F. M. (2006) '29 Si Hyperfine Structure of the E' $\alpha$ Center in Amorphous Silicon Dioxide'. Phys. Rev. Lett. 97, 135502. doi: http://bit.ly/1SIjgfe.

Dong, D., Irene, E. A., and Young, D. R. (1978) 'Preparation and Some Properties of Chemically Vapor-Deposited Si-Rich $\mathrm{SiO}_{2}$ and $\mathrm{Si}_{3} \mathrm{~N}_{4}$ Films'. J. Electrochem. Soc.: Solid-State Science and Technology, 125, 819-823. doi: 10.1149/1.2131555.

Hickmott, T. W. (1972) 'Thermoluminescence and Color Centers in rf-Sputtered Si02 Films', Journal of Applied Physics 43, 2339. doi: 10.1063/1.1661501.

López-Estopier, R., Aceves-Mijares, M., Yu, Z., and Falcony, C., (2011) 'Determination of the energy states of the donor acceptor decay emission in silicon rich oxide prepared by low-pressure chemical vapor deposition', Vacuum Science \& Technology B, Vol.29, Issue 2. doi:10.1116/1.3554735.

Pankove, J. (1971) Optical Processes in Semiconductors, Dover, New York, pp. 1718.

Richter, D. (2007) 'Advantages and Limitations of Thermoluminescence Dating of Heated Flint from Paleolithic Sites', Geoarchaeology, Vol. 22, No. 6, 671-683 (2007). doi: 10.1002/gea.20180. 
Rodríguez, J. A., Vásquez-Agustín, M. A., Morales-Sánchez, A., and Aceves-Mijares, M. (2014) 'Emission Mechanisms of Si Nanocrystals and Defects in $\mathrm{SiO}_{2}$ Materials', Journal of Nanomaterials, 409482, http://bit.ly/1NErQHj.

Salh, R. (2011a) 'Silicon nanocluster in silicon dioxide: Cathodoluminescence, energy dispersive X-ray analysis, infrared spectroscopy studies', In Crystalline Silicon Properties and Uses, (ed.) Sukumar Basu, Rijeka: InTech, pp. 173-218. doi: $10.5772 / 35404$.

Salh, R. (2011b) 'Defect related luminescence in silicon dioxide network: a review', In Crystalline Silicon - Properties and Uses, (ed.) Sukumar Basu, Rijeka: InTech, pp. 135-172. doi: 10.5772/22607.

Shinde, K. N., Dhoble, S. J., Swart, H. C., and Park, K. (2012) 'Phosphate Phosphors for Solid-State Lighting', Springer Series in Materials Science, Springer-Verlag Berlin Heidelberg, pp. 41-59.

Spallino, L., Vaccaro, L., Sciortino, L., Agnello, S., Buscarino, G., Cannas, M., and Gelardi, F. M. (2014). 'Visible-ultraviolet vibronic emission of silica nanoparticles', Phys. Chem. Chem. Phys., 16, 22028-22034. doi: 10.1039/C4CP0 2995J.

Trukhin, A. N., Troks, J., Griscom, D. L. (2007), 'Thermostimulated luminescence and electron spin resonance in X-ray- and photon-irradiated oxygen-deficient silica', Journal of Non-Crystalline Solids, 1560-1566. doi:10.1016/j.jnoncrysol. 2007.01.028.

Yacobi, B. G., and Holt, D. B. (1990) Cathodoluminescence Microscopy of Inorganic Solids, Solids Plenum, New York, 1990, pp. 43-45. 